\title{
Comment
}

\section{Sudden regime shifts after apparent stasis}

Comment on "Long transients in ecology: Theory and applications" by Andrew Morozov et al.

\section{Celso Grebogi}

grebogi@abdn.ac.uk

Institute for Complex Systems and Mathematical Biology King's College, University of Aberdeen, Aberdeen AB24 3UE, UK

Communicated by J. Fontanari

Keywords: Dynamics of ecological systems; Transient dynamics

The present review article by Andrew Morozov and co-authors is timely and highly relevant to ecological systems [1]. Long-term predictions constitute a fundamental challenge in ecology, epidemiology and climate science. Reliable forecasting is difficult because of sensitive dependence on initial conditions (the hallmark of chaos) [2], noise, and incomplete data. Another obstacle to reliable prediction of ecosystems is a phenomenon known as "regime shift" [3], where any conclusions or estimates based on the observations made before the regime shift become irrelevant after the shift. The timing of the regime shift is difficult to predict and the problem of identifying early warning signals remains largely open. Regime shift often results in a population collapse and extinction of species [4], making it an important issue for nature conservation and ecosystem management. Recently, it has been shown that regime shift can be a property of long transients - an alternative explanation that does not require any parameter change. That is, the presence of long transients may provide an alternative explanation of sudden regimes shifts which may lead to species extinction and biodiversity loss.

There are other reasons to study transient dynamics [5] in general. Traditionally, longterm dynamics have been thought to correspond to the asymptotic behaviour of the biological system, where trajectories have settled on some "final state," e.g., an equilibrium or a cycle. However, there is a growing body of empirical evidence and modelling studies showing that transient behaviour and especially long transients can play a pivotal role in the dynamics of ecosystems [6]. A similar paradigm shift occurs in epidemiology as well as other life sciences such as neuroscience and embryonic development, in climate modelling and other natural sciences. The necessity of shifting the main focus from attractors to transient dynamics lies in the very nature of biological systems: they often experience perturbations (external and/or internal) that can prevent them not only from reaching an attractor in the long-time limit but even from coming close to it. Approximating the system dynamics by considering it in the vicinity of an attractor then becomes irrelevant. Moreover, the time horizons of prediction in ecological practice and of action in ecosystem management are often considerably shorter than the relaxation time of the underlying biological system.

In spite of the importance of studying long transient dynamics, a systematic consideration of this highly relevant phenomenon in the context of life sciences is lacking. In particular, while a large number of specific examples are available, there is a lack of understanding with regard to the factors that may result in long transients, the types of the corresponding regime shifts, early warning signals, etc. Any consistent classification of transients according to the mechanisms causing them is largely missing. In particular, the important open question is what the relation, if any, is between long transients and the tipping point phenomenon.

The present review article [1] is the outcome of regular meetings and frequent video conferences of an interdisciplinary study group of ten researchers sponsored by the US 
National Science Foundation over a two-year period, addresses systematically the current issues of long transient dynamics in ecology. In particular, the study intended to partially bridge the aforementioned gaps by providing a more systematic approach to mathematical modelling of long transients in biological systems, with an emphasis on ecological applications and combining theoretical arguments with empirical observation. In doing so, the authors paid particular attention to the scaling laws of the long transients [7], i.e., how the lifetime of long transients depends on the controlling parameters of the system. The article is thus timely and highly relevant to a number of fields including ecology, epidemiology, environmental and climate sciences.

The article begins with a thorough discussion of the instructive definition of long transients in both deterministic and stochastic systems. A detailed classification scheme for the scenarios of transients in low-dimensional deterministic systems and the associated dynamical mechanisms is then presented, followed by a revisit to some conceptual mathematical models of population dynamics that exhibit long transients. The authors then describe transients in stochastic systems and emphasize the role of noise in promoting transient dynamics [8]. The impact of system complexity on transient behaviour is further considered through spatiotemporal dynamical systems and systems with a time-delay. The practically significant problem of data analysis and modelling is then addressed through a description of methods aiming to reveal transients from empirical observation with an ultimate goal of anticipating possible regime shifts. The authors conclude the article by summarizing the findings of the study group and discussing the role of transients in decision making in long-term ecosystem sustainable management. Overall, the article is well structured, quite clearly written, and friendly to a general audience with undergraduate background in mathematics, physics, and biological sciences.

While transients have been emphasized more and more in recent studies, the ecological background for the present work in fact draws on a much longer legacy. Importance of transients is inherently linked to the broader area of nonequilibrium ecological systems. A full treatment of this connection is beyond the current article, but a look at some classic papers both helps to show the development within ecology of transient thinking and emphasizes the importance of the more structured approach taken in this article. Often, the states referred to as stable are definitely not stable in a mathematical sense and this essentially means that the underlying ecological systems are best thought of as in a transient state. This clearly will have implications to policy making and management of a variety of ecosystems.

\section{Declaration of Competing Interest}

The authors declare that they have no known competing financial interests or personal relationships that could have appeared to influence the work reported in this paper.

\section{Acknowledgements}

The work is funded by the University of Aberdeen, Scotland, UK.

\section{References}

[1] A. Morozov, K. Abbott, K. Cuddington, T. Francis, G. Gellner, A. Hastings, et al., Long transients in ecology: theory and applications, Phys Life Rev 2019, https://doi.org/10.1016/j.plrev.2019.09.004.

[2] E.N. Lorenz, Deterministic nonperiodic flow, J Atmos Sci 20, 1963, 130-141.

[3] A. Morozov, M. Banerjee and S. Petrovskii, Long-term transients and complex dynamics of a stage-structured population with time delay and the Allee effect, J Theor Biol 396, 2016, 116124.

[4] J. Jiang, et al., Predicting tipping points in mutualistic networks through dimension reduction, Proc Natl Acad Sci 115, 2018, E639-E647. 
[5] Y.-C. Lai and T. Tél, Transient chaos: complex dynamics on finite-time scales, 2011, Springer; New Yorke.

[6] A. Hastings, Transient dynamics and persistence of ecological systems, Ecol Lett 4, 2001, 215220.

[7] C. Grebogi, E. Ott and J.A. Yorke, Super persistent chaotic transients, Ergod Theory Dyn Syst 5, 1985, 341-372.

[8] Y. Do and Y.-C. Lai, Scaling laws for noise-induced super persistent chaotic transients, Phys Rev E 71, 2005, 046208. 